\title{
Uniqueness of the Hamiltonian in Quantum Field Theories
}

\author{
Stephen Parrott \\ Department of Physics, University of Mass.-Boston.
}

Received June 25, 1968

\begin{abstract}
In most quantum field theories, one defines the Hamiltonian (energy) operator $H$ as a limit of "cutoff" operators $H_{s}: H=\lim _{s \rightarrow \infty} H_{s}$. (The operator $H_{s}$ would be the correct Hamiltonian for a world in which all momenta are smaller than $s$.) Since the cutoff operators seldom converge in any of the standard operator topologies, it is often necessary to invent more subtle notions of "convergence". For some of the these, it is not obvious that the "limit" operator $H$ is unique. In this note we point out that for one such method of obtaining convergence, the "limit" operator is not unique. In fact, (under mild assumptions about the operators $H_{s}$ ), if $H_{s}$ converges to $H$, then $H_{s}$ also converges to $H+R$, where $R$ is an arbitrary bounded positive operator.
\end{abstract}

\section{Notation}

Let $\mathscr{K}$ be a separable Hilbert space with inner product $(\cdot, \cdot)$. An operator $H$ on $\mathscr{K}$ is a densely-defined linear transformation from $\mathscr{K}$ into $\mathscr{K}$ with domain $\mathscr{D}(H)$. We write $H^{\prime} \subset H$ to mean $\mathscr{D}\left(H^{\prime}\right) \subset \mathscr{D}(H)$ and $H^{\prime} f=H f$ for all $f$ in $\mathscr{D}\left(H^{\prime}\right)$. A symmetric operator satisfies: $(H f, g)$ $=(f, H g)$ for all $f, g$ in $\mathscr{D}(H)$. A symmetric operator is essentially selfadjoint if it has a unique self-adjoint extension. We assume that the reader is familiar with the basic facts concerning unbounded self-adjoint operators [cf. 4, Chap. 8].

\section{Statement of the Problem}

Suppose we are given a family of self-adjoint operators $H_{s},(0<s<\infty)$. Here are two related methods for obtaining a symmetric operator $H$ as a "limit" of the family of operators $H_{s}$ :

Method $A$. Find a dense linear manifold $\mathscr{D}$ and bounded invertible operators $T_{s}, T$ such that:

(i) For all $s, T_{s} \mathscr{D} \subset \mathscr{D}\left(H_{s}\right)$.

(ii) For each $f$ in $\mathscr{K}, \lim _{s \rightarrow \infty} T_{s} f=T f$.

(iii) For all $f$ in $\mathscr{D}, \lim _{s \rightarrow \infty} H_{s} T_{s} f$ exists.

Define the limit operator $H$ with $\mathscr{D}(H)=T \mathscr{D}$ by:

$$
H T f=\lim _{s \rightarrow \infty} H_{s} T_{s} f, f \text { in } \mathscr{D} .
$$

The operator $H$ is symmetric, but need not be self-adjoint. 
Notice that, in principle, this method may yield a limit operator $H$ with $\mathscr{D}(H) \cap \mathscr{D}\left(H_{s}\right)=\{0\}$ (in which case one could not hope to obtain $\left.H f=\lim _{s \rightarrow \infty} H_{s} f\right)$.

Method $B$. Replace condition (iii) in Method A by:

(iii') For all $f, g$ in $\mathscr{D}, \lim _{s \rightarrow \infty}\left(H_{s} T_{s} f, T_{s} g\right)$ exists.

If the bilinear form defined on $T \mathscr{D} \times T \mathscr{D}$ by $T f, T g \rightarrow \lim _{s \rightarrow \infty}$ $\left(H_{s} T_{s} f, T_{s} g\right)$ is continuous in either variable, then there is a unique operator $H$ with $\mathscr{D}(H)=T \mathscr{D}$ such that $(H T f, T g)=\lim _{s \rightarrow \infty}\left(H_{s} T_{s} f, T_{s} g\right)$.

A weaker version of Method B was used in [3], and a combination of both methods was used in [1,2]. (See comment 4, Section 2.)

Notice that given $\mathscr{D}$ and $T_{s}$, the symmetric operator $H$ defined by Method A or B is unique. However, it is conceivable that one could choose a different family $T_{s}$ satisfying (i) and (ii) and obtain a different limit operator $H$. We shall see that this is, in fact, the case with Method B.

Let the family of self-adjoint operators $H_{s}$ be given. If there exists a dense linear manifold $\mathscr{D}$ and operators $T_{s}, T$ satisfying (i), (ii), and (iii) (or (iii')), we shall say that the operator $H$ defined by Method A (or B) is obtainable from $H_{s}$ by Method A (or B).

The limit operator $H$ is symmetric, but not necessarily self-adjoint, so one must pass to a self-adjoint extension of $H$ (if there is one) to obtain a self-adjoint operator as a "limit" of the operators $H_{s}$. Of course, $H$ may have more than one self-adjoint extension, so nonuniqueness can arise at this stage. However, suppose that the operator $H$ obtained from $H_{s}$ by Method A (or B) has a unique self-adjoint extension $\tilde{H}$. Let $H^{\prime}$ be another operator obtainable from $H_{s}$ by Method A. (or B). We can now pose the main question: Is $H^{\prime} \subset \tilde{H}$ ?

\section{Uniqueness of the Limit}

It is not difficult to see that an operator obtainable by Method A is unique in the sense described above. An elementary computation (see [5]) shows that if $H$ is obtainable from $H_{s}$ by Method A, then

$$
\lim _{s \rightarrow \infty}\left(H_{s}-i\right)^{-1} f=(H-i)^{-1} f \text { for all } f \text { in }(H-i) \mathscr{D}(H) .
$$

If $H$ has a unique self-adjoint extension $\tilde{H}$, then the Cayley transform $(H-i)(H+i)^{-1}$ is a unitary operator and hence $(H-i) \mathscr{D}(H)$ is dense in $\mathscr{K}$. Since the family $\left(H_{s}-i\right)^{-1}$ is uniformly bounded

$$
\lim _{s \rightarrow \infty}\left(H_{s}-i\right)^{-1} f=\left[\operatorname{cl}(H-i)^{-1}\right] f=(\tilde{H}-i)^{-1} f \text { for all } f \text { in } \mathscr{K} \text {. }
$$

If $H^{\prime}$ is also obtainable from $H_{s}$ by Method A, equation (1) holds with $H$ replaced by $H^{\prime}$. Thus $\left(H^{\prime}-i\right)^{-1} f=(\tilde{H}-i)^{-1} f$ for all $f$ in $\left(H^{\prime}-i\right) \mathscr{D}\left(H^{\prime}\right)$, and this shows that $H^{\prime} \subset \tilde{H}$. 
This result says that Method A yields a unique limit modulo the usual difficulties with uniqueness of extensions of symmetric operators. Thus the uniqueness of Method $\mathrm{A}$ is as good as one could hope. The result belows says that the uniqueness of Method $B$ is as bad as one might fear.

Theorem. Let the family $H_{s},(0<s<\infty)$ of self-adjoint operators be given, and assume that the spectrum of each $H_{s}$ contains the interval $(0, \infty)$. Suppose $H$ is obtainable from $H_{s}$ by Method $B$, and let $R$ be any bounded positive operator. Then there is an operator $H^{\prime}$ with $H^{\prime} \subset H$ such that $H^{\prime}+R$ is also obtainable from $H_{s}$ by Method $B$.

Proof. Since $H$ is obtainable from $H_{s}$ by Method $B$, we are given a dense linear manifold $\mathscr{D}$ and bounded invertible operators $T_{s}, T$, such that the conditions (i), (ii) and (iii') of Method B are satisfied. We shall show that there exists a dense linear manifold $\mathscr{D}^{\prime} \subset \mathscr{D}$ and bounded invertible operators $T_{s}$ such that:

(i) For all $s, T_{s}^{\prime} \mathscr{D}^{\prime} \subset \mathscr{D}\left(H_{s}\right)$.

(ii) $\lim _{s \rightarrow \infty} T_{s}^{\prime} f=T f$ for all $f$ in $\mathscr{K}$.

(iii') $\lim _{s \rightarrow \infty}\left(H_{s} T_{s}^{\prime} f, T_{s}^{\prime} g\right)=((H+R) T f, T g)$ for all $f, g$ in $\mathscr{D}^{\prime}$.

The operator $H^{\prime}$ will, of course, be the restriction of $H$ to $T \mathscr{D}^{\prime}$.

We shall set $T_{s}^{\prime}=T_{s}+s^{-1 / 2} U_{s} Q$, where the operators $U_{s}$ are isometries to be defined below, and $Q=(T * R T)^{1 / 2}$.

Let $P_{s}(\cdot)$ be the spectral measure associated with $H_{s}$. Then for each $f$ in $\mathscr{K}$,

$$
\lim _{\varepsilon \rightarrow 0} P_{s}((a, a+\varepsilon)) f=0 .
$$

Further, the condition that the spectrum of $H_{s}$ contains $(0, \infty)$ implies:

If $0<a<b$, then the range of $P_{s}((a, b))$ is infinite dimensional.

Let $f_{1}, f_{2}, \ldots$ be a countable set of elements in $\mathscr{D}$ whose linear span is dense in $K$. Let $\mathscr{D}^{\prime}$ be the set of all finite linear combinations of the vectors $f_{k}$. By (2), given any fixed $s$, there is a number $\varepsilon=\varepsilon(s), 0<\varepsilon(s)<1$ such that

$$
\left\|P_{s}((s, s+\varepsilon(s))) T_{s} f_{k}\right\|<\frac{1}{s} \text { for } 1 \leqq k<s .
$$

Let $E_{s}=P_{s}((s, s+\varepsilon(s)))$. By (3), the range of $E_{s}$ is infinite dimensional, and hence there exists an isometry $U_{s}$ which maps the entire Hilbert space $\mathscr{K}$ onto the range of $E_{s}$. Now

$$
\begin{aligned}
\left(H_{s} T_{s}^{\prime} f_{j}, T_{s}^{\prime} f_{k}\right)= & \left(H_{s} T_{s} f_{j}, T_{s} f_{k}\right)+s^{-1 / 2}\left(H_{s} T_{s} f_{j}, U_{s} Q f_{k}\right) \\
& +s^{-1 / 2}\left(H_{s} U_{s} Q f_{j}, T_{s} f_{k}\right)+s^{-1}\left(H_{s} U_{s} Q f_{j}, U_{s} Q f_{k}\right) .
\end{aligned}
$$

By hypothesis, the limit of the first term as $s \rightarrow \infty$ is $\left(H T f_{j}, T^{\prime} f_{k}\right)$. We shall show that the limits of the second and third terms are 0 , and 
the limit of the last term is $\left(R T f_{j}, T f_{k}\right)$. From this, the theorem follows immediately by linearity.

Note that $E_{s} U_{s}=U_{s}$ and that $E_{s}$ commutes with $H_{s}$. Thus

$$
\begin{aligned}
s^{-1 / 2}\left(H_{s} T_{s} f_{j}, U_{s} Q f_{k}\right) & =s^{-1 / 2}\left(H_{s} T_{s} f_{j}, E_{s} U_{s} Q f_{k}\right) \\
& =s^{-1 / 2}\left(H_{s} E_{s} T_{s} f_{j}, U_{s} Q f_{k}\right)
\end{aligned}
$$

The norm of the restriction of $H_{s}$ to the range of $E_{s}$ is at most $s+\varepsilon(s)$ $<s+1$, and $\mathrm{Eq}$. (4) says that for large $s,\left\|E_{s} T_{s} f_{j}\right\|<\frac{1}{s}$. Hence the modulus of $(6)$ is less than $s^{-1 / 2}(s+1)\left(\frac{1}{s}\right)\left\|Q f_{k}\right\|$, which goes to 0 as $s \rightarrow \infty$. Similarly, the limit of the third term of (5) is 0 .

As for the last term, we have

$$
\begin{aligned}
s^{-1}\left(H_{s} U_{s} Q f_{j}, U_{s} Q f_{l_{i}}\right)= & s^{-1}\left(\left(H_{s}-s\right) U_{s} Q f_{j}, U_{s} Q f_{k}\right) \\
& +\left(U_{s} Q f_{j}, U_{s} Q f_{k}\right) .
\end{aligned}
$$

Since $\left\|\left(H_{s}-s\right) U_{s}\right\| \leqq \varepsilon(s)<1$, the first term of $(7)$ tends to 0 as $s \rightarrow \infty$. And, $\left(U_{s} Q f_{j}, U_{s} Q f_{k}\right)=\left(Q f_{j}, Q f_{k}\right)=\left(R T f_{j}, T f_{k}\right)$ because $U_{s}$ is an isometry.

\section{Comments}

1. The hypothesis that the spectrum of $H_{s}$ contains $(0, \infty)$ simplifies the proof, but is stronger than is necessary. It is sufficient to assume that there exist numbers $m(s)$ with $\lim _{s \rightarrow \infty} m(s)=\infty$ such that $P_{s}((m(s), m(s)+1))$ has infinite-dimensional range. Stated loosely, the only case in which the $H_{s}$ are unbounded and the conclusion of the theorem may be false is when some of the $H_{s}$ have spectrum whose unbounded part consists of a discrete sequence of eigenvalues of finite multiplicity. In this case, one can show that the limit is never unique, but it is probably not true that the conclusion of the theorem holds.

2. There are examples in which the conclusion of the theorem holds with $\mathscr{D}^{\prime}=\mathscr{D}$. This is true even if $\mathscr{D}$ has uncountable Hamel dimension.

3. It is worth noting that in case the operators $H_{s}$ are uniformly bounded, the convergence defined by Method A (respectively, B) is actually convergence in the strong (resp. weak) operator topology.

4. In [1], the author defines the renormalized Hamiltonian $H_{\text {ren }}$ as a limit (in the sense of Method B) of the renormalized cutoff operators $H_{\text {ren }}(s)$. The nonuniqueness of Method B noted above indicates that this procedure is undesirable, and we shall briefly describe here how one can obtain the renormalized Hamiltonian of [1] without recourse to Method B. All page numbers refer to [1].

The operators $H_{\text {ren }}(s)$ can be written as a sum $H_{\text {ren }}(s)=H_{1 s}+V_{2 s}$ (pages $344,369,383$ ). The author proves:

(a) $H_{1 s}$ converges in the sense of Method A to an operator $H_{1}$ (p. 381). 
(b) There is an operator $V_{2}$ such that $\mathscr{D}\left(H_{1}\right) \subset \mathscr{D}\left(V_{2}\right)$ (p. 382, 383, 358 ) and for $g_{1}, g_{2}$ in $\mathscr{D}\left(H_{1}\right), \lim _{s \rightarrow \infty}\left(g_{1}, V_{2 s} g_{2}\right)=\left(g_{1}, V_{2} g_{2}\right)$ (p. 383). Thus $H_{1}$ and $V_{2}$ are both defined on $\mathscr{D}\left(H_{1}\right)$, and each is a (unique) limit of the corresponding cutoff operators $H_{1 s}, V_{2 s}$.

It is natural to define the renormalized Hamiltonian $H_{\text {ren }}$ by: $H_{\text {ren }}=H_{1}+V_{2}$.

I wish to thank Professor JaMes GLIm for many helpful and stimulating conversations.

\section{References}

1. GLIMm, J.: Yukawa coupling of quantum fields in two dimensions, I. Commun. Math. Phys. 5, 343 (1967).

2. - Yukawa coupling of quantum fields in two dimensions, II. Commun. Math. Phys. 6, 61 (1967).

3. - Boson fields with the : $\Phi^{4}$ : interaction in three dimensions, Commun. Math. Phys. (to appear).

4. Riesz, F., and Sz-NAGY, B. : Functional analysis. New York: Fred. Ungar 1955.

5. Glimm, J., and JAffee, A.: Singular perturbations of self adjoint operators. Comm. Pure Appl. Math. Phys. (to appear).

S. K. Parrott

Department of Mathematics

University of Massachusetts at Boston

Boston, Mass. 02116, USA 